\title{
Endovascular treatment of femoro-popliteal artery stenosis/obstruction using a repositionable self-expanding nitinol stent: a preliminary study
}

\author{
Seyhan Yılmaz', Behice Kaniye Yılmaz², Özgür Barış', Orhan Fındık', Mehmet Kalender', Eray Aksoy ${ }^{3}$ \\ 'Derince Education and Research Hospital, Department of Cardiovascular Surgery, Kocaeli, Turkey \\ ${ }^{2}$ Corum Education and Research Hospital, Department of Radiology, Corum, Turkey \\ ${ }^{3}$ American Hospital, Department of Cardiovascular Surgery, Istanbul, Turkey
}

\begin{abstract}
Introduction. The aim of this study was to report our preliminary results regarding the use of the repositionable self-expanding nitinol stent in a cohort of patients with femoro-popliteal artery occlusion/stenosis.

Material and methods. The study groups consisted a total of 8 patients ( 10 extremities) between 47 and 70 years of age who presented with typical symptoms of intermittent claudication and/or critical limb ischaemia (Fontaine stage II-IV) and underwent primary elective superficial femoral artery percutaneous transluminal angioplasty and/or stenting using a Jaguar repositionable self-expanding nitinol stent in the dates of 20 I3-2015. Information for patency at 6th month after the stenting procedure was obtained.

Results. The mean operation time was $38.56 \pm 26.40$ minutes. No patients had procedure-related stent fracture, distal thromboembolism, haematoma, femoral pseudoaneurysm or stent occlusion. At 6-month follow-up, at least one categorical improvement was observed in 7 patients (87.5\% of intent-to-treat population). Conclusions. The Jaguar self-expanding nitinol stent has several technical advantages, and its use was associated with a low risk of complications. Further study is warranted to establish its superiority in providing good long-term patency.
\end{abstract}

Key words: peripheral obstructive disease, angioplasty, critical limb ischaemia

Acta Angiol 2015; 21, 4: II6-121

\section{Introduction}

Lower extremity peripheral artery disease (PAD) is a major health problem leading to significant morbidity and even mortality. As a result of the evolving knowledge regarding the multi-systemic nature of atherosclerotic disease, PAD is no longer considered to be a problem limited to the extremity involved, but has increasingly been recognized as a distinct entity requiring a multifaceted approach including interventional treatment [I]. As the disease course may be asymptomatic until stenosis reaches an advanced stage, PAD is commonly underdiagnosed and undertreated [2]. In the last decade, the total number of PAD cases was reported to have increased by $13.1 \%$ to $28.7 \%$ worldwide, with the most marked increases in prevalence observed among patients with advanced disease and those living in low-income countries [3]. Although recent advances in diagnostic modalities and increasing use of endovascular techniques have led to a significant decrease in amputation rates, mortality has remained unchanged in patients hospitalized for PAD [4].

Patients with superficial femoral artery stenosis make up an important proportion of patients with $P A D$, and since this type of involvement was reported to be most commonly associated with intermittent claudication, this patient population has been subject to intensive research on methods to prevent disease

Address for correspondence: Seyhan Yilmaz, Derince Education and Research Hospital, Kocaeli, Turkey, tel.: +905336838I86, fax: +902622230323, e-mail: drlabarna@gmail.com 
progression and further complications [5]. Although endovascular revascularization is the recommended and preferred treatment in patients with single superficial femoral artery stenosis shorter than $10 \mathrm{~cm}$ or single occlusion shorter than $5 \mathrm{~cm}$ Trans-Atlantic Inter-Society Consensus [TASC] A or in those having multiple stenosed or occluded segments shorter than $5 \mathrm{~cm}$ (TASC type B), whether primary stenting achieves better outcomes than primary transluminal angioplasty in patients with superficial femoral artery (SFA) lesions has remained controversial $[6,7]$.

Recently, there has been an increasing trend towards the initial use of endovascular stents in the treatment of SFA lesions. However, despite their suggested technical advantages, balloon expandable stainless steel stents did not demonstrate a superior sustained benefit over percutaneous transluminal angioplasty. This outcome has led to the development of second-generation, self-expanding nitinol stents that are more flexible and thus more resistant [8]. The Jaguar SM stent (Balton, Warsaw, Poland) is a novel self-expanding nitinol stent featuring a unique deployment system that allows correction of the stent position even after the partial release of the stent inside the arterial lumen. A recent study that evaluated its usefulness in patients with iliac artery disease reported promising results [9]. However, to our knowledge, there has been no study reporting the use of the Jaguar stent for the treatment of superficial femoral artery lesions.

Here we report our preliminary results regarding the use of the Jaguar self-expanding stent in a cohort of patients with TASC type A-C stenosis.

\section{Material and methods}

The study was approved by the local ethics committee. All patients were informed about the planned procedure and provided informed consent. This retrospective cohort study was undertaken in two tertiary hospitals and a total of 39 patients (mean age $63.42 \pm$ 9.23 years) undergoing endovascular peripheral arterial interventions for PAD between the dates of 20I3-20I 5 . A total of 8 patients ( 10 extremities) between 47 and 70 years of age (mean age $59.40 \pm 7.32$ years) who presented with typical symptoms of intermittent claudication and/or critical limb ischaemia (Fontaine stage II-IV) and underwent primary elective SFA percutaneous transluminal angioplasty (PTA) and stenting using a Jaguar repositionable self-expanding nitinol stent in the dates of 2013-2015 were considered eligible for the study.

All patients were initially evaluated with duplex ultrasound scanning, and those with positive findings indicating diminished arterial flow to the lower extrem- ity underwent computed tomography (CT) angiography to reveal the degree and extent of the stenosis.

Patients were considered eligible if they had TASC type $A$ (single stenosis $<10 \mathrm{~cm}$ in length or single occlusion $<5 \mathrm{~cm}$ in length) or TASC type B (multiple lesions $<5 \mathrm{~cm}$ or single stenosis $<15 \mathrm{~cm}$ not involving the popliteal artery) or TASC type $C$ (multiple stenosis or occlusions totalling $>15 \mathrm{~cm}$ with or without heavy calcification) superficial femoral artery lesions [6]. Patients were excluded if they had unilateral or bilateral aorto-iliac stenosis or occlusion, chronic total occlusion in the common or superficial femoral artery $(>20 \mathrm{~cm}$ involving the popliteal artery) or popliteal artery or underwent to primary elective PTA only. Patients with diffuse peripheral arterial involvement and those with a clear indication for concomitant or staged vascular procedures were also excluded. Patients with unstable coronary symptoms and those with a high risk profile for coronary artery disease were not included, and instead referred for cardiovascular investigation, whereas asymptomatic patients with a low risk profile for coronary artery disease did not undergo coronary angiography.

Patient demographics, counselling charts, and laboratory findings were recorded. Pre-procedural CT angiography evaluation was performed by a radiologist who was unaware of patients. All of the patients had PAD. Smoker patients were encouraged to undertake smoking cessation and patients who had high LDL levels (> $100 \mathrm{mg} / \mathrm{dL}$ ) were initiated on statins. Hypertensive patients were treated appropriately and diabetic patients underwent consultation with an endocrine and diabetes specialist to control blood sugar levels before the procedure. Patients who had a previous history of coronary artery disease underwent consultation with a cardiologist for necessity of coronary angiography before the procedure.

\section{Procedure}

In all patients, acetylsalicylic acid $100 \mathrm{mg} /$ day and clopidogrel $75 \mathrm{mg} /$ day were initiated on the same day of the procedure. All procedures were performed in a vascular surgery operating room under local anaesthesia and with the aid of fluoroscopy guidance using a portable X-ray machine. All procedures were performed by the same vascular surgeons.

With the patient in a supine position, skin antisepsis was performed with povidone iodine, followed by the placement of sterile surgical drapes. A 5-6 Fr introducer catheter was placed in the ipsilateral common femoral artery and advanced from the groin distally in a downward direction. Intra-arterial injection of 5000 IU heparin was followed by preliminary angiography to reveal the stenosis. The procedure proceeded in a percutaneous fashion, according to the manufacturer's instructions. 
A guidewire was inserted through the introducer catheter using either a subintimal angioplasty or intraluminal angioplasty technique. During the subintimal technique, predilatation was performed after re-entry to the intraluminal space was achieved. A self-expanding nitinol stent delivery sheath was introduced over the guidewire and advanced beyond the distal end of the lesion. The stent position was checked fluoroscopically, and the system allowed for accurate stent placement before deployment by re-positioning in the upward and downward direction based on serial fluoroscopic images. Finally, the stent was released, and the delivery system was removed. Post-dilatation was performed when necessary. The introducer was removed, and the bleeding was controlled by manual compression (Fig. I).

After the procedure, patients were transferred to the vascular surgery ward and discharged on the following day. Distal pulses were examined before the discharge, and the patients were invited to a follow-up examination 6 months after the procedure. Follow up included clinical assessment and physical examination of the patient. Doppler ultrasound imaging was performed to check for stent patency if the patients were symptomatic or distal lower extremity pulses were weak or absent.

\section{Statistical analysis}

Statistical analyses were performed using MedCalc statistical software. Normal distribution of the parameters was assessed using visual histograms and the Shapiro-Wilk test. Continuous parameters were expressed as mean \pm standard deviation, and categorical parameters as numbers and percentages within brackets. A p value less than 0.05 was considered statistically significant.
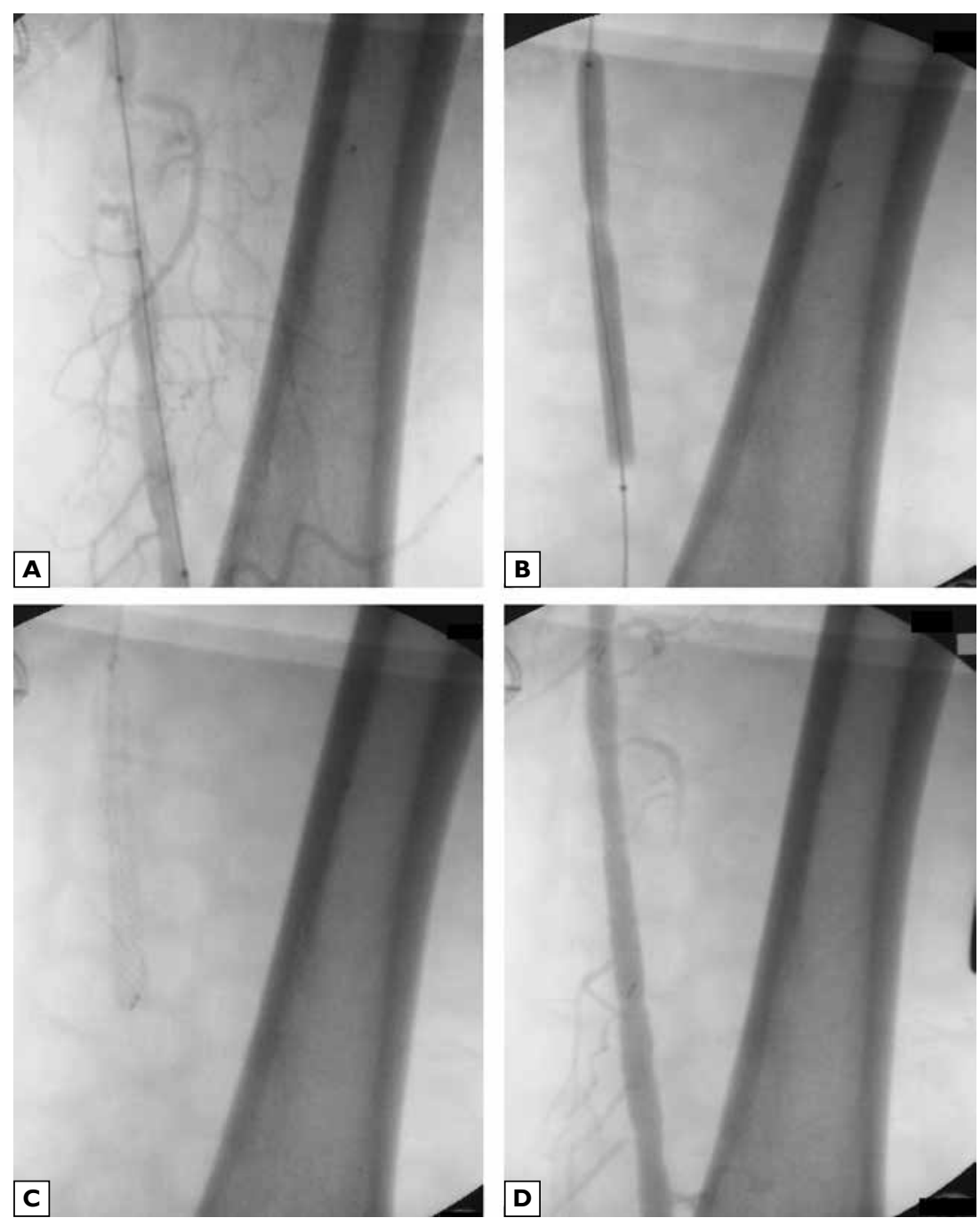

Figure I. Representative fluoroscopic images during peripheral artery stenting; A. Guidewire advanced beyond the lesion within the superficial femoral artery; B. Predilatation; C. Stent deployed; D. Post-procedural view 
Table I. Baseline characteristics of intent-to-treat patients $(\mathrm{N}=8)$

\begin{tabular}{|l|c|}
\hline Variable & Mean \pm SD or N (\%) \\
\hline Age (years) & $59.40 \pm 7.32$ \\
\hline Males & $8(100 \%)$ \\
\hline Clinical presentation & \\
\hline Fontaine IIb & $5(62.5 \%)$ \\
\hline Fontaine III & $2(25.0 \%)$ \\
\hline Fontaine IV & $\mathrm{I}(12.5 \%)$ \\
\hline Diabetes & $5(62.5 \%)$ \\
\hline Smoking & $7(87.5 \%)$ \\
\hline Hypertension & $5(62.5 \%)$ \\
\hline Dyslipidaemia & $4(50.0 \%)$ \\
\hline Stable coronary artery disease & $4(50.0 \%)$ \\
\hline
\end{tabular}

Table 2. Distribution of TASC II lesional involvement in intent-to-treat extremities $(\mathrm{N}=10)$

\begin{tabular}{|l|c|}
\hline \multicolumn{2}{|c|}{$\mathbf{N}(\%)$} \\
\hline Type A lesions & $7(70.0 \%)$ \\
\hline Type B lesions & $2(20.0 \%)$ \\
\hline Type C lesions & $1(10.0 \%)$ \\
\hline Total & 10 \\
\hline
\end{tabular}

\section{Results}

The age of the study subjects was between 47 and 70 years of age (mean age $59.40 \pm 7.32$ years) and all were males. Five patients $(62.5 \%)$ patients ( 7 extremities) had severe claudication ( $<200 \mathrm{~m}$ walking distance, Fontaine stage IIb). Critical limb ischaemia, defined as the presence of ischaemic rest pain (Fontaine stage III) or ulcers or gangrene (Fontaine stage IV) lasting for more than 2 weeks, was present in 3 patients (37.5\%). Mean lesion length was $4.3 \pm 3.22 \mathrm{~cm}$ and eight (80\%) of the lesions were stenoses. Baseline patient characteristics are shown in Table I and the distribution of TASC II lesional involvement in patients' extremities is included in Table 2.

Mean operation time was $38.56 \pm 26.40$ minutes, and mean amount of contrast used was $50.36 \pm 27.17 \mathrm{~mL}$ during the procedure. Unilateral stenting was performed in 6 patients, and bilateral stenting was performed in 2 patients. Thus, there were a total of 8 intent-to-treat patients and 10 intent-to-treat extremities. The procedural information regarding intent-to-treat extremities is included in Table 3 . The procedure was successfully completed in all patients, and no patients required any additional intervention or conversion to open surgery.
Table 3. Procedural information regarding intent-to-treat extremities $(\mathrm{N}=10)$

\begin{tabular}{|l|c|}
\hline Variable & N \\
\hline Technique & 2 \\
\hline $\begin{array}{l}\text { Stenting via subintimal angio- } \\
\text { plasty }\end{array}$ & 8 \\
\hline $\begin{array}{l}\text { Stenting via intraluminal angio- } \\
\text { plasty }\end{array}$ & 6 \\
\hline Location & 2 \\
\hline SFA stenting & 2 \\
\hline Popliteal artery stenting & $\begin{array}{l}\text { Both SFA and popliteal artery } \\
\text { stenting }\end{array}$ \\
\hline
\end{tabular}

SFA — superficial femoral artery

During the procedure, repositioning of the stent was performed in $2(20.0 \%)$ extremities to avoid occluding large collateral side-branches originating quite close to the proximal or distal end of the stenotic lesion.

None of the patients had procedure-related complications and complications on follow-up like stent fracture, distal thromboembolism, haematoma, femoral pseudoaneurysm, or early stent occlusion. One patient who initially had Fontaine IV symptoms and type $C$ stenosis and underwent unilateral stenting was re-hospitalized with acute worsening of lower extremity ischaemic symptoms I month after the procedure. CT angiography revealed $95 \%$ in-stent stenosis, and this patient underwent a surgery.

All remaining patients completed 6-month follow-up. Clinical assessment revealed that 4 of 5 patients with Fontaine stage Ilb PAD shifted to Fontaine stage Ila, one of 5 patients with Fontaine stage Ilb PAD shifted to Fontaine stage I, and all 2 patients with Fontaine stage III PAD shifted to Fontaine stage IIb. One patient with Fontaine stage IV PAD did not complete 6-month follow-up as described above. Thus, at least one categorical improvement was observed in 9 extremities ( $90.0 \%$ of the intent-to-treat population). In the follow-up period, the superficial femoral artery stent was patent in all per-protocol extremities $(90.0 \%$ of intent-to-treat extremities).

\section{Discussion}

In this preliminary study, we achieved satisfactory outcomes using the Jaguar repositionable self-expanding nitinol stent for the treatment of superficial femoral artery stenosis or occlusions. The technique provided excellent post-procedural outcomes and had a low rate of complications. Also, given the acceptable operative 
times we obtained, it offers a steep learning curve for the operator through its easy implantation technique. The delivery system of the device is flexible and allows for advancing through tortuous arterial segments, although this feature was not of apparent use to us during our practice since the superficial femoral artery follows a straighter course within the thigh when compared to the aorto-iliac system. Nevertheless, none of our patients had stent-related mechanical complications such as fracture or displacement, which is attributable to the basic feature of the stent itself being made of a single nitinol wire without welds and solders, thus providing further elasticity.

Currently, endovascular therapy is the treatment of choice in patients with single short segment stenosis or occlusion (type A lesions), and it is also recommended in the presence of multiple short-segment stenoses (type B lesions) [6]. However, whether implantation of stents provides better outcomes when compared with PTA alone is still controversial [10]. Several studies have compared stents with various treatment options. In an earlier meta-analysis, Balk and colleagues [ $\mathrm{II}]$ found no significant difference in clinical outcomes including re-intervention, amputation, mortality, and complication rates between patients receiving either PTA or stenting. In a randomized trial, Kedora et al. [12] compared Viabahn stent grafting and bypass surgery for the treatment of SFA occlusion. Similar to our study, their patients had an adequate distal vascular bed and were also free of aorto-iliac occlusive disease. Clinical improvement, limb salvage and mortality did not differ at the end of follow-up.

Nevertheless, recent data has indicated more promising results regarding the effectiveness of additional techniques in the treatment of infrainguinal peripheral artery disease. In a recent meta-analysis including a total 40 randomized trials, Simpson et al. [13] sought to determine whether patients undergoing endovascular treatment for femoro-popliteal artery stenosis/occlusion benefited from additional techniques including self-expanding stents, drug-coated balloons, and drug-eluting stents. The authors reported that self-expanding stents were associated with a lower restenosis rate at 6 months and also that stent grafting significantly reduced restenosis compared to percutaneous transluminal angioplasty alone. A more recent meta-analysis revealed that nitinol stents were associated with superior technical success, whereas drug-eluting stents provided better long-term patency in femoro-popliteal artery lesions [14]. Our preliminary experiences with the self-expanding Jaguar nitinol stent support the technical superiority of nitinol stenting; the procedure was quite simple in that we were able to perform it without C-arm fluoroscopy but rather with the aid of a portable X-ray machine. The procedure was performed in a vascular surgery operating room since a hybrid revascularization room has not yet been established in our institution.

Primary nitinol stenting is preferable for the treatment of femoro-popliteal artery occlusive disease, despite conflicting results regarding its long-term benefit $[15,16]$. In biomechanical studies, stents in the femoro-popliteal artery were shown to be subject to compression and bending by articular movement, which was postulated to lead to stent failure in the long term [ I 7]. Moreover, bare nitinol stenting was shown to be associated with high rates of restenosis. These findings have led to the development of numerous new stents to increase graft patency survival $[18,19]$. Among them, the Supera helical interwoven stent stands out for its enhanced flexibility to resist against fracture when placed in the femoro-popliteal artery. Early experience using this type of stent was promising, with primary patency rates of $83.5 \%$ and $78.6 \%$ at 6 - and 12 -month follow-up, respectively [20]. The technical features of this stent are similar to that used in the present study. Given that none of the patients in this previous cohort, similar to ours, had stent fractures during follow-up, newer stents seem to provide more promising longterm outcomes compared to those used in the past.

Our preliminary study had several limitations including a non-randomized design, lack of control group and low number of patients. At follow up, Doppler ultrasonography was only done when patients had symptoms or when physical examination revealed poor distal flow. Angiographic evaluation was performed only in those patients having signs suggestive of stent occlusion in Doppler assessment. Thus, follow up data lacked objective imaging data to draw a conclusion regarding the patency rate of the stent used in this study and also its influence on disease progression.

\section{Conclusions}

Currently there is no ongoing international study registered in clinicaltrials.gov regarding the use of self-expanding Jaguar nitinol stents. Our preliminary study concluded that this newer stent has several technical advantages, and its use was also associated with a low risk of complications. Further study is warranted to establish its superiority in providing good long-term patency, as has been proposed based on its enhanced flexibility and resistance against bending forces. 


\section{References}

I. Paraskevas KI, Mukherjee D, Whayne TF Jr. (2013) Peripheral arterial disease: implications beyond the peripheral circulation. Angiology; 64: 569-57I.

2. Argyriou C, Saleptsis V, KoutsiasS, Giannoukas AD (2013) Peripheral arterial disease is prevalent but underdiagnosed and undertreated in the primary care setting in central Greece. Angiology; 64: I19-124.

3. Fowkes FG, Rudan D, Rudan I et al (2013) Comparison of global estimates of prevalence and risk factors for peripheral artery disease in 2000 and 2010: a systematic review and analysis. Lancet; 382: 1329-1340.

4. Malyar N, Fürstenberg T, Wellmann J et al (20/3) Recent trends in morbidity and in-hospital outcomes of in-patients with peripheral arterial disease: a nationwide population-based analysis. Eur Heart J; 34: 2706-2727.

5. White $C$ (2007) Intermittent claudication. N Engl J Med; 356: |24|-1250.

6. Norgren L, Hiatt WR, Dormandy JA, Nehler MR, Harris KA, Fowkes FGR (2007) Inter-Society Consensus for the Management of Peripheral Arterial Disease (TASC II). Eur J Vasc Endovasc Surg; 33: SI-S75.

7. Twine CP, Coulston J, ShandallA, McLain AD (2009) Angioplasty versus stenting for superficial femoral artery lesions. Cochrane Database Syst Rev; 2: CD006767.

8. Litsky J, Chanda A, Stilp E, Lansky A, Mena C (2014) Critical evaluation of stents in the peripheral arterial disease of the superficial femoral artery - focus on the paclitaxel eluting stent. Med Devices (Auckl); 7: 149-156.

9. Kordecki K, Lukasiewicz A, Nowicki M et al (2012) Assessment of effectiveness of endovascular treatment of common and external iliac artery stenosis/occlusion using self-expanding Jaguar SM stents. Pol J Radiol; 77: 22-29.

10. Health Quality Ontario (2010) Stenting for peripheral artery disease of the lower extremities: an evidence-based analysis. Ont Health Technol Assess Ser; 10: 1-88.

II. Balk E, Cepeda MS, Ip S, Trikalinos T, O'Donnell T (2008) Horizon scan of invasive interventions for lower extremity peripheral artery disease and systematic review of studies comparing stent placement to other interventions [Internet]. Rockville, MD: Agency for Healthcare Research and Quality. Report No.: ARTS0407.

12. Kedora J, Hohmann S, Garrett W, Munschaur C, Theune B, Gable D (2007) Randomized comparison of percutaneous Viabahn stent grafts vs prosthetic femoral-popliteal bypass in the treatment of superficial femoral arterial occlusive disease. J Vasc Surg; 45: 10-16.

13. Simpson EL, Michaels JA, Thomas SM, Cantrell AJ (2013) Systematic review and meta-analysis of additional technologies to enhance angioplasty for infrainguinal peripheral arterial occlusive disease. Br J Surg; 100: I I 28-1 I 37.

14. Katsanos K, Spiliopoulos S, Karunanithy N, Krokidis M, Sabharwal T, Taylor P (20/4) Bayesian network meta-analysis of nitinol stents, covered stents, drug-eluting stents, and drug-coated balloons in the femoropopliteal artery. J Vasc Surg; 59: I | 23- I 333.

15. AcinF, de Haro J, Bleda S, Varela C, Esparza L (2012) Primary nitinol stenting in femoropopliteal occlusive disease: a meta-analysis of randomized controlled trials. J Endovasc Ther; 19: 585-595.

16. Sakamoto $Y$, Hirano K, lida $O$ et al. (2013) Five-year outcomes of self-expanding nitinol stent implantation for chronic total occlusion of the superficial femoral and proximal popliteal artery. Catheter Cardiovasc Interv; 82: E25I-E256.

17. Early M, Kelly DJ (20II) The consequences of the mechanical environment of peripheral arteries for nitinol stenting. Med Biol Eng Comput; 49: 1279-1288.

18. Rocha-Singh KJ, Jaff MR, Crabtree TR, Bloch DA, Ansel G (2007) Performance goals and endpoint assessments for clinical trials of femoropopliteal bare nitinol stents in patients with symptomatic peripheral arterial disease. Catheter Cardiovasc Interv; 69: 910-919.

19. Banerjee S, Das TS, Abu-Fadel MS et al (2012) Pilot trial of cryoplasty or conventional balloon post-dilation of nitinol stents for revascularization of peripheral arterial segments: the COBRA trial. J Am Coll Cardiol; 60: 1352-1359.

20. Aghel A, Armstrong EJ (2014) Recent advances in self-expanding stents for use in the superficial femoral and popliteal arteries. Expert Rev Cardiovasc Ther; 12: 833-842. 